\title{
Hepatic resection after neoadjuvant chemotherapy for patients with liver metastases from colorectal cancer: need for cautious planning
}

\author{
Young II Kim¹, In Ja Park', Jeong Eun Kim², So Yeon Kim³, Jin-Hong Park', Jae Hoon Lee ${ }^{5}$, Tae Yong Ha ${ }^{6}$, \\ Yong Sang Hong ${ }^{2}$, Sun Young Kim², Tae Won Kim², Seok-Byung Lim', Chang Sik Yu', Jin Cheon Kim ${ }^{1}$ \\ ${ }^{1}$ Department of Colon and Rectal Surgery, Asan Medical Center, University of Ulsan College of Medicine, Seoul, Korea \\ ${ }^{2}$ Department of Oncology, Asan Medical Center, University of Ulsan College of Medicine, Seoul, Korea \\ ${ }^{3}$ Department of Radiology, Asan Medical Center, University of Ulsan College of Medicine, Seoul, Korea \\ ${ }^{4}$ Department of Radiation Oncology, Asan Medical Center, University of Ulsan College of Medicine, Seoul, Korea \\ ${ }^{5}$ Division of Hepatobiliary and Pancreatic Surgery, Asan Medical Center, University of Ulsan College of Medicine, Seoul, Korea \\ ${ }^{6}$ Division of Hepatobiliary Surgery and Liver Transplantation, Asan Medical Center, University of Ulsan College of Medicine, Seoul, \\ Korea
}

Purpose: Current neoadjuvant chemotherapy (NAC) may enable therapies such as surgical resection and local ablation of metastases in patients with colorectal liver metastasis (CLM). We evaluated outcomes in CLM patients who underwent resection and/or local treatment after NAC and identified prognostic factors for oncologic outcomes.

Methods: Patients who received NAC followed by resection and/or local treatment of hepatic metastasis from 2013 to 2015 were included. Treatment and tumor-related variables were tabulated. Recurrence-free survival (RFS) and overall survival (OS) were analyzed using the Kaplan-Meier method. Cox regression analysis was used to identify factors associated with RFS and OS.

Results: Sixty-eight patients received NAC followed by resection and/or local treatment of hepatic metastases. Targeted therapy was administered in $50 \%$ of the patients. RFS was $35.8 \%$ at 1 year and $19.4 \%$ at 2 years postoperatively. OS was $95.6 \%$ at 1 year and $88.2 \%$ at 2 years postoperatively. In the multivariable analysis, R1 resection margin (hazard ratio [HR], 3.95; $P=0.008)$ of the liver metastases and ypN1/ypN2 (HR, 2.356 and 1.983, respectively; $P=0.041)$ were associated with poor RFS. Both factors were also significantly related to OS.

Conclusion: Resection margin of the metastatic tumor and ypN status are the only relevant factors for RFS and OS in CLM patients treated with NAC. Despite early and high rates of recurrence, CLM patients treated with NAC who undergo resection and/or local treatment have acceptable OS. Multidisciplinary review of candidates for surgery and cautious planning are crucial for achieving optimal outcomes.

[Ann Surg Treat Res 2019;97(5):245-253]

Key Words: Colorectal cancer, Liver metastases, Neoadjuvant chemotherapy, Survival outcome

Received July 31, 2019, Revised September 3, 2019,

Accepted October 5, 2019

Corresponding Author: In Ja Park

Department of Colon and Rectal Surgery, Asan Medical Center, University of Ulsan College of Medicine, 88 Olympic-ro 43-gil, Songpa-gu, Seoul 05505, Korea

Tel: +82-2-3010-3937, Fax: +82-2-474-9027

E-mail: ipark@amc.seoul.kr

ORCID: https://orcid.org/0000-0001-5355-3969
Copyright (c) 2019, the Korean Surgical Society

(c) Annals of Surgical Treatment and Research is an Open Access Journal. All articles are distributed under the terms of the Creative Commons Attribution NonCommercial License (http://creativecommons.org/licenses/by-nc/4.0/) which permits unrestricted non-commercial use, distribution, and reproduction in any medium, provided the original work is properly cited. 


\section{INTRODUCTION}

Over the recent years, survival rates of patients receiving initial hepatectomy for liver metastases have markedly improved. Patients with untreated colorectal liver metastases (CLMs) have a poor median survival of 4.5 months (range, 0-36 months) [1]. Therefore, aggressive treatments including resection are increasingly performed in CLM patients, when possible [2]. Also, with the improvement of chemotherapy in the last decade, patients with liver metastases that are considered unresectable may be reassessed as resectable after neoadjuvant chemotherapy (NAC) [3]. However, recurrence of liver metastasis after hepatectomy is still very common (up to $50 \%$ ), and studies report associations of recurrence with inferior overall survival (OS) and other outcomes [4].

Searching for factors related to survival or recurrence is important. Knowing such information beforehand can help clinicians and patients choose the most beneficial treatment. Several studies have identified features such as CLM size, number of CLMs, number of NAC cycles, response to NAC, and other clinical/morphological factors relevant to patient oncologic outcomes [5-7]. Contradicting studies suggest that the response to NAC does not predict the OS of patients with CLM and that salvage treatment may benefit patients with poor response to NAC [8]. Several studies report that patients with positive resection margins of the resected CLM having markedly worse outcomes compared to those whose resected CLMs have clear margins. CLM resection remains a favored approach despite studies reporting that nearly $10 \%$ of patients undergoing CLM resection have CLMs with R1 margins on pathologic examination [9-12]. Additionally, biological features such as KRAS mutation are known to be associated with poor outcomes [13].

These various study results must be translated into clinical action; more information is needed to manage each patient according to the most appropriate customized treatment. The clinical considerations for planning CLM treatment after NAC remain to be clarified. To investigate the prognostic factors for oncologic outcomes in CLM patients, we evaluated the outcomes of colorectal cancer patients with liver metastasis who underwent resection and/or local treatment after NAC.

\section{METHODS}

\section{Patients}

The study population was derived from a historical cohort of consecutive patients with colorectal cancer who underwent hepatic resection and/or local treatment for CLM from January 2013 to December 2015 at Asan Medical Center, Seoul, Korea. Colorectal cancer was diagnosed according to the World Health Organization International Classification and staged according to the American Joint Committee on Cancer manual, seventh edition [14]. All patients underwent digital rectal examination (DRE), blood tests including measurement of CEA concentrations, colonoscopy with biopsy, chest radiography, and CT scanning of the abdomen; pelvic MRI or transrectal ultrasound was done for patients with mid- or low-rectal cancer. Survival status, NAC regimens, clinicopathological characteristics of the primary tumor, clinicopathological features of liver metastasis, and type of surgical or local treatment were obtained retrospectively from the patients' medical records. Formalin-fixed paraffin-embedded tissues were prepared for extended KRAS and BRAF testing by high throughput sequencing (OncoMap ver. 4.0).

Patients with synchronous colorectal cancer and liver metastasis were included in this study, regardless whether the metastases were unresectable, marginally resectable, or resectable. Resectability was determined by a multidisciplinary team consisting of colorectal surgeons, oncologists, radiation oncologists, and at least 2 radiologists with a subspecialty in gastroenterology. Patients who did not receive NAC were excluded. All patients underwent resection of the primary colorectal tumor with surgical and/or interventional treatment for liver metastasis. Patients who received only palliative resection of the primary tumor were excluded. Of the total of 293 patients who underwent liver resection and/or local treatment for synchronous CLM, after exclusion of 203 patients who did not receive NAC, 21 patients with palliative colorectal tumor resection, and 1 lost to follow-up, 68 patients with CLMs resected with curative intent who received NAC were included (Fig. 1).

The study protocol was approved by the Institutional Review Board (IRB) of Asan Medical Center (approval number: S20191139-0001) and informed consent was waived according to IRB regulations.

\section{Treatment of colorectal cancer and liver metastasis}

Patients received NAC with FOLFIRI (folinic acid, fluorouracil, and irinotecan); FOLFOX (folinic acid, fluorouracil, and oxaliplatin)/XELOX (capecitabine and oxaliplatin); or LF (folinic acid, fluorouracil), with or without targeted agents (bevacizumab or cetuximab). Surgery was conducted 4 to 6 weeks after the last administration of chemotherapy. Surgery for patients with severe side effects of chemotherapy was delayed until recovery. Primary tumor and liver metastases were both resected with curative intent. Local treatment for liver metastasis consisted of radiofrequency ablation (RFA) or stereotactic body radiation therapy (SBRT). Patients with co-morbidity and patients at risk of inadequate remnant liver volume after resection received local treatments. SBRT was administered to patients with a total of 48 Gy unit in 4 fractions. All RFA was performed intraoperatively by an interventional radiologist. Real-time 


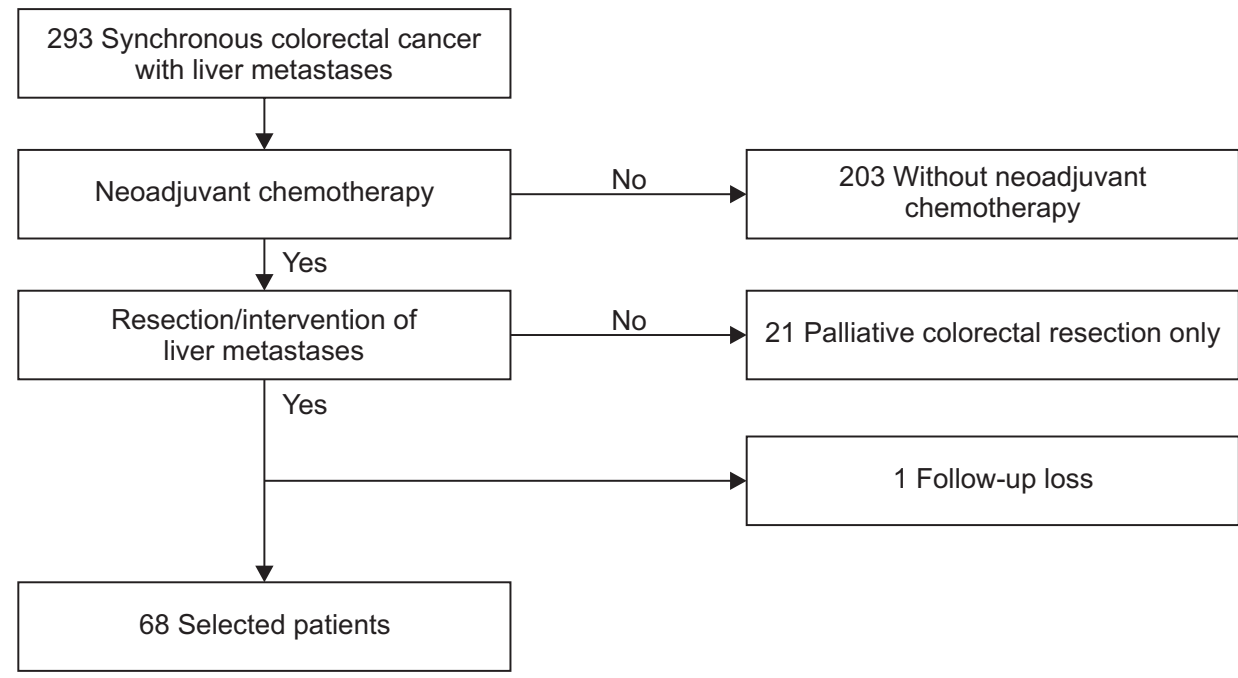

Fig. 1. Patient selection flowchart. ultrasonography guidance was used with a single internally cooled electrode. A maximum output of $200 \mathrm{~W}$ was delivered to the center of the metastatic tumor for 10 minutes. Result of ablation was evaluated immediately after the procedure with intraoperative ultrasonography.

Follow-up examinations including DRE, complete blood count, liver function test, serum CEA concentration, and chest radiography were performed every 3 months for the first 2 years after surgery and every 6 months thereafter. Abdominal and pelvic CT was performed every 3 months and chest CT every 6 months. Colonoscopy was performed 1 year after surgery and every 3 years thereafter. Tumor recurrence was defined as progressive soft-tissue growth and hypermetabolic lesions detected by $\mathrm{CT}$. The radiologic images were primarily reviewed by gastrointestinal radiologists.

\section{Statistical analysis}

Primary outcomes were recurrence-free survival (RFS) and OS. RFS was defined as the time interval from the initial resection to the first documented detection of recurrent disease on CT during regular follow-up. OS was defined as the time from initial resection to the date of death from any cause.

Rates of RFS and OS were estimated using the Kaplan-Meier method. Multivariable analysis with the Cox proportional hazards model was used to compare risk factors associated with RFS and OS. Cutoff values, calculated using receiver operating characteristic curve analysis, were 58 years for age and $3 \mathrm{~cm}$ for tumor size. Variables were included in the multivariable analysis (total cycles of NAC, largest CLM size before NAC, ypN categories, perineural invasion, liver resection margin, and number of CLMs after NAC). A P-value less than 0.05 was considered statistically significant. Patients with incomplete data were included in the analysis. All statistical analyses were performed using IBM SPSS Statistics ver. 21.0 (IBM Co., Armonk,
NY, USA).

\section{RESULTS}

\section{Clinicopathological characteristics of patients and primary tumors \\ Of the 68 patients, $49(72.1 \%)$ were male. Targeted agents were} used in 34 patients $(50 \%)$. The location of the primary tumor was right colon in 4 patients (5.9\%), left colon in 20 (29.4\%), rectum in $43(63.2 \%)$, and synchronous in $1(1.5 \%)$. Twentyseven patients (39.7\%) were ypN0, 28 patients (41.2\%) ypN1, and 13 patients (19.1\%) were ypN2. Lymphovascular invasion and perineural invasion were present in 29 patients $(42.6 \%)$ and 26 patients (38.2\%) respectively. Sixteen patients (23.5\%) had KRAS mutation. The total median follow-up period was 41 months (interquartile range [IQR], 27.00-49.75). The clinicopathological characteristics of all 68 patients are shown in Table 1.

\section{Characteristics of liver metastases}

Before receiving NAC, 26 patients (38.2\%) had CLM in one lobe of the liver and 42 patients $(61.8 \%)$ had bilobular CLM. After NAC, 33 patients (47.8\%) had unilobular CLM and 35 patients (52.2\%) still had bilobular lesions. Median tumor size of the largest CLM was $3.8 \mathrm{~cm}$ before NAC and $2.2 \mathrm{~cm}$ after NAC. Before NAC, 26 patients (38.2\%) had tumor size smaller than $3 \mathrm{~cm}$ and 42 patients (61.8\%) had tumors larger than $3 \mathrm{~cm}$. After NAC, 44 patients (64.7\%) had tumor size smaller than 3 $\mathrm{cm}$ and 24 patients (35.3\%) had tumors larger than $3 \mathrm{~cm}$. The median number of liver metastases before and after NAC was 5 (IQR, 2-8) and 3 (IQR, 1-4), respectively.

Sixty-five patients were treated with resection for CLM and 3 patients received local treatments (RFA, SBRT). According to metastatic tumor locations, partial hepatectomy was performed in 35 patients: right lobectomy in 6 patients, left lobectomy in 6 
Table 1. Clinicopathological characteristics $(n=68)$

\begin{tabular}{|c|c|}
\hline Characteristics & Value \\
\hline Age (yr) & $57.6 \pm 11.9$ \\
\hline \multicolumn{2}{|l|}{ Sex } \\
\hline Male & $49(72.1)$ \\
\hline Female & $19(27.9)$ \\
\hline \multicolumn{2}{|l|}{ Total cycles of NAC } \\
\hline$\leq 4$ & $28(41.2)$ \\
\hline$\geq 5$ & $40(58.8)$ \\
\hline \multicolumn{2}{|l|}{ Type of NAC } \\
\hline FOLFOX/XELOX & $18(26.5)$ \\
\hline FOLFIRI & $4(5.9)$ \\
\hline Capecitabine & $6(8.8)$ \\
\hline LF & $6(8.8)$ \\
\hline FOLFOX/FOLFIRI + cetuximab & $22(32.4)$ \\
\hline FOLFOX/FOLFIRI + bevacizumab & $12(17.6)$ \\
\hline Targeted agent use & $34(50)$ \\
\hline \multicolumn{2}{|l|}{ Location of primary tumor } \\
\hline Right colon & $4(5.9)$ \\
\hline Left colon & $20(29.4)$ \\
\hline Rectum & $43(63.2)$ \\
\hline Synchronous & $1(1.5)$ \\
\hline \multicolumn{2}{|l|}{ урT categories } \\
\hline $0-2$ & $9(13.2)$ \\
\hline $3-4$ & $59(86.8)$ \\
\hline \multicolumn{2}{|l|}{ ypN categories } \\
\hline 0 & $27(39.7)$ \\
\hline 1 & $28(41.2)$ \\
\hline 2 & $13(19.1)$ \\
\hline Lymphovascular invasion & $29(42.6)$ \\
\hline Perineural invasion & $26(38.2)$ \\
\hline \multicolumn{2}{|l|}{ KRAS } \\
\hline Wild & $36(52.9)$ \\
\hline Mutant & $16(23.5)$ \\
\hline Not available & $16(23.5)$ \\
\hline \multicolumn{2}{|l|}{ BRAF } \\
\hline Wild & $49(74.2)$ \\
\hline Mutant & $1(1.5)$ \\
\hline Not available & $18(24.2)$ \\
\hline
\end{tabular}

Values are presented as mean \pm standard deviation or number $(\%)$. NAC, neoadjuvant chemotherapy; FOLFIRI, folinic acid, fluorouracil, and irinotecan; FOLFOX, folinic acid, fluorouracil, and oxaliplatin; XELOX, capecitabine and oxaliplatin; LF, folinic acid, fluorouracil.

patients, right posterior segmentectomy in 2 patients, and left lateral sectionectomy in 2 patients. Fourteen patients received partial hepatectomy combined with any segmentectomy. Resected CLMs of 59 patients (86.8\%) had pathological R0 resection margins and lesions from 6 patients $(8.8 \%)$ had R1 resection margins (Table 2). The resection margins of the 3 patients treated with local therapy alone (2 RFA, and 1 SBRT) could not be assessed. Sixty-five patients (95.6\%) received adjuvant chemotherapy. Three patients could not receive further treatment due to poor general condition.
Table 2. Clinicopathologic characteristics of liver metastases $(\mathrm{n}=68)$

\begin{tabular}{lc}
\hline \multicolumn{1}{c}{ Characteristics of liver metastases } & Value \\
\hline Location of lesions before NAC & \\
Unilobular & $26(38.2)$ \\
Bilobular & $42(61.8)$ \\
Location of lesions after NAC & \\
Unilobular & $33(47.8)$ \\
Bilobular & $35(52.2)$ \\
Largest tumor size (cm) before NAC ${ }^{\text {a) }}$ & \\
s3 & $26(38.2)$ \\
$>3$ & $42(61.8)$ \\
Largest tumor size (cm) after NAC ${ }^{\text {a) }}$ & \\
$\quad \leq 3$ & $44(64.7)$ \\
$>3$ & $24(35.3)$ \\
Total number of liver metastases before NAC & $5(2-8)$ \\
Total number of liver metastases after NAC & $3(1-4)$ \\
Type of treatments & \\
Resection only & $58(85.3)$ \\
Resection + local therapy & $7(10.3)$ \\
Local therapy only & $3(4.4)$ \\
Resection margin & \\
R0 & $59(86.8)$ \\
R1 & $6(8.8)$ \\
No resection & $3(4.4)$ \\
\hline
\end{tabular}

Values are presented as number (\%) or median (interquartile range).

NAC, neoadjuvant chemotherapy; local therapy includes radiofrequency ablation and stereotactic brachy radiotherapy.

${ }^{\mathrm{a})}$ Size measured on radiologic images.

The median postoperative hospital stay was 8 days (IQR, 7.25-10 days). Postoperative complication was noted in 13 patients (19.1\%). Ileus in 5 patients, pleural effusion in 2 patients, elevated amylase/lipase in 2 patients, abdominal fluid collection in 1 patient, bladder injury in 1 patient, superior mesenteric vein thrombosis in 1 patient, and 1 wound complication. There was no 30-day mortality.

\section{Oncologic outcomes and factors associated with oncologic outcome}

The median RFS was 8.0 months and the median OS was 50.0 months (Fig. 2). For patients with R0 and R1 resection margins, the median RFS was 8 and 4.4 months, respectively $(P=0.008)$ and OS was 52.0 months and 27.0 months, respectively $(\mathrm{P}=$ 0.003). For ypN0, ypN1, and ypN2, the median RFS was 13.2, 6.6, and 7.1 months, respectively $(\mathrm{P}=0.029)$, and median OS was 65 , 42, and 50 months, respectively ( $\mathrm{P}=0.022$ ) (Fig. 3).

Fifty-six patients (82.5\%) experienced recurrence after primary treatment of CLM. The median time to recurrence after surgery was 7 months. The most common recurrence site was the liver (25 patients, 36.8\%). Fourteen patients (20.6\%) had recurrence in the lung. Eight patients $(11.8 \%$ ) had recurrence 

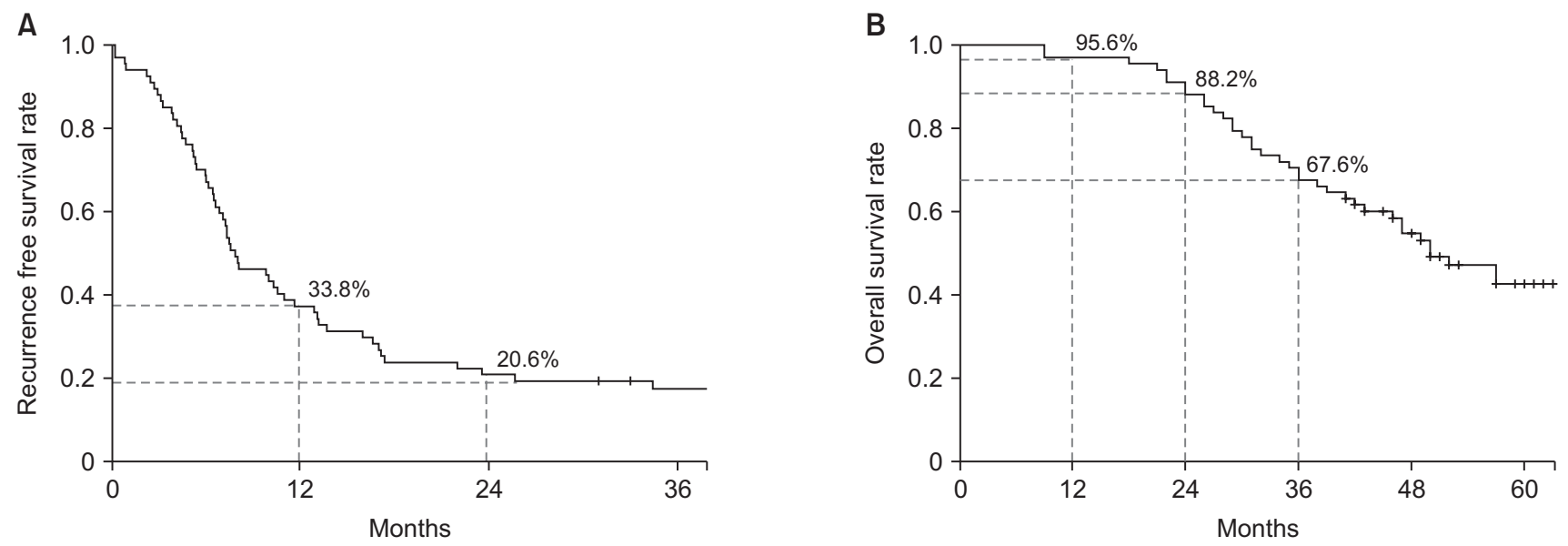

Fig. 2. Kaplan-Meier analysis of survival rates. Recurrence-free survival (A) and overall survival (B) for the 68 patients with colorectal cancer and liver metastasis who received surgical and/or local therapy after neoadjuvant chemotherapy.
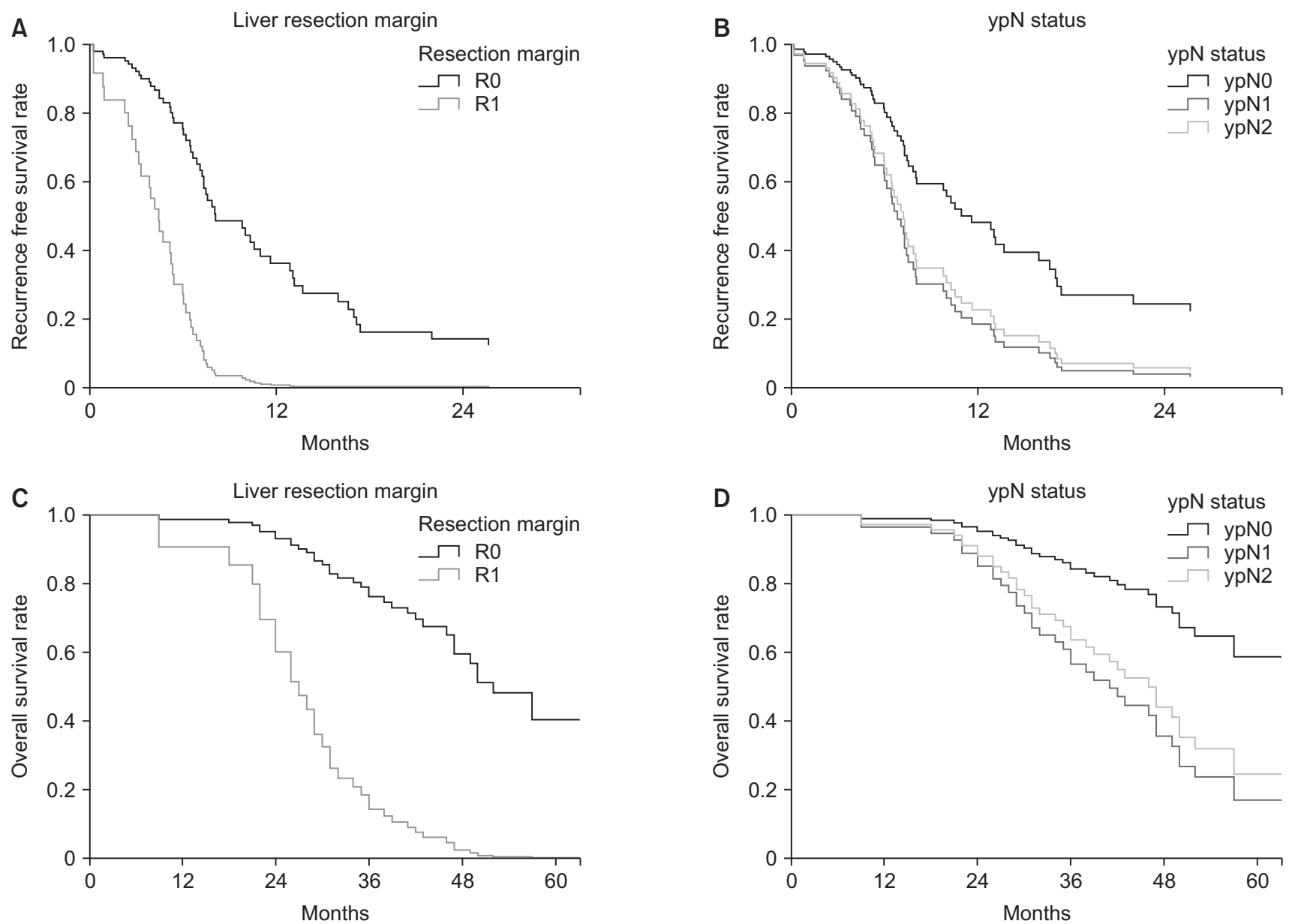

Fig. 3. Cox proportional hazards model of oncologic outcomes in patients with different resection margin and different ypN status. (A) Recurrence-free survival (RFS) according to resection margin. (B) RFS according to ypN status. (C) Overall survival (OS) according to resection margin. (D) OS according to ypN status.

in the lung and liver simultaneously. Lymph node recurrence occurred in 2 patients (2.9\%). One patient had recurrence in the ovary and 1 patient had recurrence in the anastomosis site of prior surgery. Recurrence was treated with chemotherapy alone (30 patients, 44.1\%), local treatment (surgical resection, RFA, and SBRT; 6 patients, 8.8\%) alone, and both local treatment and 
chemotherapy (19 patients, 27.9\%). Treatment of recurrence was not recorded in 1 patient who was lost to follow up. Recurrence was significantly associated with poor OS $(\mathrm{P}<0.001)$.

Univariate analysis showed that more than 5 total cycles of NAC (HR, 2.40; $P=0.003$ ), largest CLM size before NAC $>3 \mathrm{~cm}$ (HR, 1.97; $\mathrm{P}=0.013$ ), $\mathrm{R} 1$ resection margin (HR, 5.80; $\mathrm{P}<0.001$ ), and number of CLMs after NAC $\geq 3$ (HR, 3.13; $\mathrm{P}<0.001$ ) were significantly associated with patient RFS. The multivariable analysis results showed that $\mathrm{R} 1$ resection margin $(\mathrm{HR}, 3.95 ; \mathrm{P}=$
0.008 ) and ypN1, 2 categories (HR, 2.356 and 1.983 respectively; $P=0.041$ ) were significantly related to patient RFS (Table 3). In the identical settings, $\mathrm{R} 1$ resection margin (HR, 4.701; $\mathrm{P}=0.003)$ and ypN1, ypN2 status (HR, 2.137; P = 0.027 and $\mathrm{HR}, 2.630 ; \mathrm{P}=$ 0.019 , respectively) were both significantly associated with poor OS.

Table 3. Results of Cox proportional hazards analysis to identify factors associated with recurrence-free survival

\begin{tabular}{|c|c|c|c|c|}
\hline \multirow{2}{*}{ Variable } & \multicolumn{2}{|c|}{ Univariable analysis } & \multicolumn{2}{|c|}{ Multivariable analysis } \\
\hline & Hazard ratio $(95 \% \mathrm{Cl})$ & P-value & Hazard ratio $(95 \% \mathrm{Cl})$ & P-value \\
\hline Age $>58$ yr & $0.91(0.53-1.55)$ & 0.719 & & \\
\hline \multicolumn{5}{|l|}{ Sex } \\
\hline Female & 1.00 (reference) & & & \\
\hline Male & $0.794(0.44-1.44)$ & 0.448 & & \\
\hline \multicolumn{5}{|l|}{ Total cycles of NAC } \\
\hline$\leq 4$ & 1.00 (reference) & & 1.00 (reference) & \\
\hline$\geq 5$ & $2.396(1.35-4.25)$ & 0.003 & $1.425(0.689-2.950)$ & 0.339 \\
\hline Targeted agent use as NAC & $1.068(0.628-1.817)$ & 0.809 & & \\
\hline Adjuvant chemotherapy & $3.094(0.427-22.432)$ & 0.264 & & \\
\hline \multicolumn{5}{|l|}{ Largest CLM size $(\mathrm{cm})$ before NAC } \\
\hline$\leq 3$ & 1.00 (reference) & & 1.00 (reference) & \\
\hline$>3$ & $1.973(1.151-3.381)$ & 0.013 & $1.375(0.723-2.614)$ & 0.332 \\
\hline \multicolumn{5}{|l|}{ Largest CLM size $(\mathrm{cm})$ after NAC } \\
\hline$\leq 3$ & 1.00 (reference) & & & \\
\hline$>3$ & $1.450(0.817-2.573)$ & 0.205 & & \\
\hline \multicolumn{5}{|l|}{ урт categories } \\
\hline урт0-2 & 1.00 (reference) & & & \\
\hline урT2-4 & $1.482(0.632-3.474)$ & 0.366 & & \\
\hline ypN categories & & 0.057 & & 0.041 \\
\hline ypNO & 1.00 (reference) & & 1 (reference) & \\
\hline ypN1 & $1.987(1.088-3.628)$ & 0.025 & $2.356(1.203-4.611)$ & \\
\hline ypN2 & $1.989(0.940-4.210)$ & 0.072 & $1.983(0.855-4.599)$ & \\
\hline Lymphovascular invasion-positive & $1.331(0.781-2.267)$ & 0.293 & & \\
\hline Perineural invasion-positive & $1.628(0.95-2.791)$ & 0.076 & $1.073(0.583-1.974)$ & 0.822 \\
\hline \multicolumn{5}{|l|}{ Liver resection margin } \\
\hline R0 & 1.00 (reference) & & 1.00 (reference) & \\
\hline $\mathrm{R} 1$ & $5.804(2.211-15.233)$ & $<0.001$ & $3.949(1.435-10.864)$ & 0.008 \\
\hline \multicolumn{5}{|l|}{ KRAS status } \\
\hline Wild & 1.00 (reference) & & & \\
\hline Mutant & $1.278(0.680-2.402)$ & 0.445 & & \\
\hline \multicolumn{5}{|l|}{ BRAF status } \\
\hline Wild & 1.00 (reference) & & & \\
\hline Mutant & $0.719(0.098-5.269)$ & 0.745 & & \\
\hline \multicolumn{5}{|l|}{ No. of CLM before NAC } \\
\hline $0-2$ & 1.00 (reference) & & & \\
\hline$\geq 3$ & $1.618(0.890-2.941)$ & 0.115 & & \\
\hline \multicolumn{5}{|l|}{ No. of CLM after NAC } \\
\hline $0-2$ & 1.00 (reference) & & 1.00 (reference) & \\
\hline$\geq 3$ & $3.127(1.766-5.538)$ & $<0.001$ & $1.911(0.943-3.869)$ & 0.072 \\
\hline
\end{tabular}

All factors were checked at the time of primary surgery.

$\mathrm{Cl}$, confidence intervals; CLM, colorectal liver metastasis; NAC, neoadjuvant chemotherapy. 


\section{DISCUSSION}

In this study, involving a pre-selected group of patients with CLM who received NAC at a single tertiary center, we sought to identify relevant factors for oncologic outcomes; the results show that resection margin of the metastatic tumor and ypN status are the only relevant factors for RFS and OS. Patients with hepatic resection margin involvement and lymph node metastases from the primary tumor experienced poor oncologic outcomes; namely, recurrence and OS.

Despite the significant survival outcome difference between patients with $\mathrm{R} 0$ and $\mathrm{R} 1$ margin tumors, both groups still have a very favorable OS compared to patients with untreated CLM. Recent studies, which included similar patient groups, all reported short disease-free intervals but favorable OS (Table 4). These studies reported less than 1 year, approximately, of RFS (similar to the median RFS of 8.0 months in the present study) with $66.2 \%$ of patients experiencing recurrence within 1 year after surgery. As summarized in Table 4, these studies, and the present study, all report an OS of more than 4 years, indicating that even with the high risk of recurrence, active treatment for CLM patients can yield acceptable OS outcomes.

Surgeons face a dilemma in determining the timing of surgery after NAC to achieve optimal oncologic outcomes in their patients. When the surgery is scheduled too late, it may be difficult for surgeons to locate the tumor in patients with a good response to NAC. Also, patients may experience severe complications of chemotherapy, jeopardizing their postoperative condition and making surgeons hesitant to perform surgery according to protocol. Additionally, patients may be at risk of a poor response to chemotherapy, with disease progression preventing an oncologically sound resection. In the present study, the operation was scheduled to occur 1 month after NAC, and the actual median time interval from the end of NAC to the date of operation was 5.0 weeks (IQR, 4-7 weeks). Surgery was postponed due to reasons mentioned above and as resection is the only treatment for cure, cautious planning and adjusting treatment agenda considering individual patient conditions by a multidisciplinary team approach is critical.

Although most studies report consistent findings of early recurrence but a relatively favorable OS in CLM patients after resection, the factors associated with RFS or OS have differed. Factors are assessed either independently or in a scoring system for their relation to oncologic outcomes. Clinical risk scores (CRS) are used to determine the prognosis of patients with CLM after resection; widely used scoring systems have been developed by Fong et al. [15] Nordlinger et al. [2], Nagashima et al. [16], and Konopke et al. [17]. The different CRS consist of common factors such as pathologic $\mathrm{T}$ stage, pathologic lymph node stage, number of liver metastases, and size of the largest CLM, with slight variations. However, despite their wide used, the CRS may be outdated, as the scoring systems were established over a decade ago and most CRS excluded patients with R1 resections. CLM patients who have undergone resection may present with R1 margins even though they were assessed as curatively resectable prior to surgery; thus, determining patient prognosis without accounting for potential R1 resections can be challenging.

A clear resection margin of the metastatic hepatic tumor is important for achieving a favorable oncologic outcome $[10,11,18,19]$. Traditionally, a $1-\mathrm{cm}$ rule was followed to provide a safe margin width [20-22], but recently, many studies have reported that the margin width might not be as crucial $[10,23]$. Accordingly, pathologic reports from the present study defined an R0 margin as a clear margin regardless of the width of normal tissue secured, without considering a safety margin. Even though the results of this and previous studies show significantly poor outcomes in cases with R1 margins, the reason for this difference may be complex. An R1 margin may indicate residual tumor in the patient, but it may also represent

Table 4. Publications describing survival outcomes of resection for colorectal liver metastasis after neoadjuvant chemotherapy

\begin{tabular}{|c|c|c|c|c|}
\hline Study & $\begin{array}{l}\text { No. of } \\
\text { patients }\end{array}$ & $\begin{array}{l}\text { Recurrence-free } \\
\text { survival (mo) }\end{array}$ & OS (mo) & Relevant factors of RFS and/or OS \\
\hline Present study & 68 & 7.87 & 50.0 & CLM resection margin, ypN state \\
\hline Serayssol et al., 2019 [27] & 150 & $14 \%$ (3-yr RFS rate) & $57 \%$ (3-yr OS rate) & $\begin{array}{l}\text { Response to NAC, number of NAC } \\
\text { cycles }\end{array}$ \\
\hline Wimmer et al., 2017 [28] & 227 & 9 & 50 & CRS (Nagahima score) \\
\hline Ayez et al., 2012 [24] & 92 & 16 & 65 & T stage, Node state \\
\hline Ayez et al., 2011 [29] & 159 & 9 & 47 & CRS (Nordlinger score) \\
\hline Gallagher et al., 2009 [8] & 111 & $\mathrm{NR}$ & 62 & CLM size, CLM resection margin \\
\hline Wein et al., 2003 [30] & 20 & $52 \%(2-y r$ RFS rate $)$ & $\begin{array}{l}80 \% \text { ( } 2 \text {-yr cancer-related } \\
\text { survival rate })\end{array}$ & $\mathrm{NR}$ \\
\hline
\end{tabular}

OS, overall survival; RFS, recurrence-free survival; CLM, colorectal liver metastasis; NAC, neoadjuvant chemotherapy; CRS, clinical risk score; NR, not recorded. 
a more aggressive tumor that is challenging to remove radically. Therefore, it is important for surgeons and other members of the multidisciplinary team to identify the patients most likely to have lesions with an R0 resection margin. Multiple tumors and bilateral distribution are the most common factors associated with R1 margins [9,18,23]; the results of the present study show the correlation of R1 margins with multiple tumors (total number of CLM after NAC, P $=0.021$ ) but not with bilateral distribution (location of lesions after NAC, $\mathrm{P}=0.543$ ). According to these results, patients with multiple (more than 3) tumors after NAC must be evaluated for resectability more meticulously and cautiously.

In contrast, one study specifically indicated that an R1 margin is not predictive for RFS and OS in patients who had received NAC [24]; no difference in RFS and OS was found between patients with $\mathrm{R} 0$ and $\mathrm{R} 1$ resections. That study reported 18 months of RFS in patients with $\mathrm{R} 0$ resections versus 9 months of RFS in those with R1 resections $(P=0.303)$ and 65 months of OS in patients with R0 resections versus those with R1 resections in whom the median OS was not reached $(P=0.645)$. They speculated that NAC could have destroyed the micrometastases in the periphery; therefore, patients had the same survival rate regardless of the width of the negative margin. These results contradict the results of this and many other previous studies. One major difference in the study that did not identify R1 margins as a relevant factor was that none of the patients in that study received adjuvant chemotherapy after CLM surgery. In the present study, $95.6 \%$ of the patients received adjuvant chemotherapy after CLM surgery. This difference in patient treatment may be the reason for the discrepancy between the studies. Further research is needed to verify the correlation.

The results of the present study show a significant association between recurrence and survival outcomes, similar to the results of prior studies $[4,25]$. Recurrence was most common in the liver, followed by lung. The 2 most common recurrence sites frequently presented as oligometastatic. This feature is important in managing recurrent disease, as the current guidelines emphasize treatment of oligometastatic diseases [26]. Patients with a single site recurrence, either local or distant (liver, lung), may benefit from a complete resection of the recurrent tumor [25]. Of the 56 patients with recurrence in the present study, 17 patients (30.4\%) underwent surgical resection with or without systemic treatment. We did not compare survival outcomes between patients with recurrence who received surgery and those who did not, but the fact that nearly $1 / 3$ of the recurred patients with recurrence were candidates for curative resection is encouraging. Also, the advancement of interventions such as RFA and SBRT may benefit patients with a single recurrence that is unresectable due to a difficult location or the patient's general condition.

The present study is limited by its retrospective, nonrandomized design. Patient information was collected by the provider and was not standardized within the single center. Also, there was no baseline control group with which to compare and assess the benefits of NAC or resection of CLM.

In conclusion, resection after NAC for patients with CLM, when possible, offers the chance of long-term survival, but careful assessment is essential for determining patient prognosis and altering treatment plans. Various factors among all reported predictors of RFS and OS, not only resection margin and node status, should be considered. Trials involving multiple institutions are needed to advance the comprehensive understanding of this disease and further improve patients' oncologic outcomes.

\section{CONFLICTS OF INTEREST}

No potential conflict of interest relevant to this article was reported.

\section{REFERENCES}

1. Bengtsson G, Carlsson G, Hafstrom L, Jonsson PE. Natural history of patients with untreated liver metastases from colorectal cancer. Am J Surg 1981;141:5869.

2. Nordlinger B, Guiguet M, Vaillant JC, Balladur P, Boudjema K, Bachellier P, et al. Surgical resection of colorectal carcinoma metastases to the liver. A prognostic scoring system to improve case selection, based on 1568 patients. Association Française de Chirurgie. Cancer 1996;77: 1254-62.

3. Bismuth H, Adam R, Levi F, Farabos C, Waechter F, Castaing D, et al. Resection of nonresectable liver metastases from colorectal cancer after neoadjuvant chemotherapy. Ann Surg 1996;224:509-20.

4. Hallet J, Sa Cunha A, Adam R, Goere D, Bachellier P, Azoulay D, et al. Factors influencing recurrence following initial hepatectomy for colorectal liver metastases. Br J Surg 2016;103:1366-76.

5. Allen PJ, Kemeny N, Jarnagin W, DeMatteo R, Blumgart L, Fong Y. Importance of response to neoadjuvant chemotherapy in patients undergoing resection of synchronous colorectal liver metastases. J Gastrointest Surg 2003;7:109-17.

6. Adam R, Pascal G, Castaing D, Azoulay D, 
Delvart V, Paule B, et al. Tumor progression while on chemotherapy: a contraindication to liver resection for multiple colorectal metastases? Ann Surg 2004:240: 1052- 61.

7. Gomez D, Cameron IC. Prognostic scores for colorectal liver metastasis: clinically important or an academic exercise? HPB (Oxford) 2010;12:227-38.

8. Gallagher DJ, Zheng J, Capanu M, Haviland D, Paty P, Dematteo RP, et al. Response to neoadjuvant chemotherapy does not predict overall survival for patients with synchronous colorectal hepatic metastases. Ann Surg Oncol 2009;16:1844-51.

9. de Haas RJ, Wicherts DA, Flores E, Azoulay D, Castaing D, Adam R. R1 resection by necessity for colorectal liver metastases: is it still a contraindication to surgery? Ann Surg 2008;248:626-37.

10. Poultsides GA, Schulick RD, Pawlik TM. Hepatic resection for colorectal metastases: the impact of surgical margin status on outcome. HPB (Oxford) 2010;12: 43-9.

11. Vigano L, Procopio F, Cimino MM, Donadon M, Gatti A, Costa G, et al. Is tumor detachment from vascular structures equivalent to R0 resection in surgery for colorectal liver metastases? An observational cohort. Ann Surg Oncol 2016;23: 1352-60.

12. Margonis GA, Buettner S, Andreatos N, Sasaki K, Ijzermans JNM, van Vugt JLA, et al. Anatomical resections improve disease-free survival in patients with KRAS-mutated colorectal liver metastases. Ann Surg 2017;266:641-9.

13. Cucchetti A, Russolillo N, Johnson P, Tarchi P, Ferrero A, Cucchi M, et al. Impact of primary cancer features on behaviour of colorectal liver metastases and survival after hepatectomy. BJS Open 2018;3:18694.

14. Edge SB, Compton CC. The American Joint Committee on Cancer: the 7th edition of the AJCC cancer staging manual and the future of TNM. Ann Surg Oncol 2010;17:1471-4.

15. Fong Y, Fortner J, Sun RL, Brennan MF,
Blumgart LH. Clinical score for predicting recurrence after hepatic resection for metastatic colorectal cancer: analysis of 1001 consecutive cases. Ann Surg 1999; 230:309-18.

16. Nagashima I, Takada T, Adachi M, Nagawa H, Muto T, Okinaga K. Proposal of criteria to select candidates with colorectal liver metastases for hepatic resection: comparison of our scoring system to the positive number of risk factors. World J Gastroenterol 2006;12:6305-9.

17. Konopke R, Kersting S, Distler M, Dietrich J, Gastmeier J, Heller A, et al. Prognostic factors and evaluation of a clinical score for predicting survival after resection of colorectal liver metastases. Liver Int 2009:29:89-102.

18. Nuzzo G, Giuliante F, Ardito F, Vellone M, Giovannini I, Federico B, et al. Influence of surgical margin on type of recurrence after liver resection for colorectal metastases: a single-center experience. Surgery 2008;143:384-93.

19. Jung SW, Kim DS, Yu YD, Han JH, Suh SO. Risk factors for cancer recurrence or death within 6 months after liver resection in patients with colorectal cancer liver metastasis. Ann Surg Treat Res 2016;90: 257-64.

20. Wakai T, Shirai Y, Sakata J, Valera VA, Korita PV, Akazawa K, et al. Appraisal of 1 $\mathrm{cm}$ hepatectomy margins for intrahepatic micrometastases in patients with colorectal carcinoma liver metastasis. Ann Surg Oncol 2008;15:2472-81.

21. Wray CJ, Lowy AM, Mathews JB, Park S, Choe KA, Hanto DW, et al. The significance and clinical factors associated with a subcentimeter resection of colorectal liver metastases. Ann Surg Oncol 2005;12: 374-80.

22. Cady B, Jenkins RL, Steele GD Jr, Lewis WD, Stone MD, McDermott WV, et al. Surgical margin in hepatic resection for colorectal metastasis: a critical and improvable determinant of outcome. Ann Surg 1998;227:566-71.

23. Figueras J, Burdio F, Ramos E, Torras J, Llado L, Lopez-Ben S, et al. Effect of sub- centimeter nonpositive resection margin on hepatic recurrence in patients undergoing hepatectomy for colorectal liver metastases. Evidences from 663 liver resections. Ann Oncol 2007;18:1190-5.

24. Ayez N, Lalmahomed ZS, Eggermont AM, Ijzermans JN, de Jonge J, van Montfort K, et al. Outcome of microscopic incomplete resection (R1) of colorectal liver metastases in the era of neoadjuvant chemotherapy. Ann Surg Oncol 2012;19:1618-27.

25. Weiser MR, Jarnagin WR, Saltz LB. Colorectal cancer patients with oligometastatic liver disease: what is the optimal approach? Oncology (Williston Park) 2013; 27:1074-8.

26. Van Cutsem E, Cervantes A, Adam R, Sobrero A, Van Krieken JH, Aderka D, et al. ESMO consensus guidelines for the management of patients with metastatic colorectal cancer. Ann Oncol 2016;27: 1386-422.

27. Serayssol C, Maulat C, Breibach F, Mokrane FZ, Selves J, Guimbaud R, et al. Predictive factors of histological response of colorectal liver metastases after neoadjuvant chemotherapy. World J Gastrointest Oncol 2019;11:295-309.

28. Wimmer K, Schwarz C, Szabo C, Bodingbauer M, Tamandl D, Mittlbock M, et al. Impact of neoadjuvant chemotherapy on clinical risk scores and survival in patients with colorectal liver metastases. Ann Surg Oncol 2017:24:236-43.

29. Ayez N, Lalmahomed ZS, van der Pool AE, Vergouwe $\mathrm{Y}$, van Montfort K, de Jonge J, et al. Is the clinical risk score for patients with colorectal liver metastases still useable in the era of effective neoadjuvant chemotherapy? Ann Surg Oncol 2011; 18:2757-63.

30. Wein A, Riedel C, Bruckl W, Merkel S, Ott R, Hanke B, et al. Neoadjuvant treatment with weekly high-dose 5-Fluorouracil as 24-hour infusion, folinic acid and oxaliplatin in patients with primary resectable liver metastases of colorectal cancer. Oncology 2003;64:131-8. 